\title{
Kinematics and Dynamics of $f(R)$ Theories of GRAVITY
}

\author{
Steve Rippl ${ }^{1 *}$ Henk van Elst ${ }^{1 \dagger}$, Reza TAVAKol ${ }^{1 \ddagger} \&$ David TAYlor ${ }^{2,3 \S}$ \\ ${ }^{1}$ Astronomy Unit, School of Mathematical Sciences, Queen Mary $\&$ Westfield College, Mile End Road \\ London E1 $4 N S, U K$ \\ ${ }^{2}$ Department of Computational and Applied Mathematics \\ University of the Witwatersrand, PO WITS, 2050, South Africa \\ ${ }^{3}$ School of Mathematical Studies, University of Portsmouth, PO1 2EG, UK
}

May 3, 1995

\begin{abstract}
We generalise the equations governing relativistic fluid dynamics given by Ehlers and Ellis for general relativity, and by Maartens and Taylor for quadratic theories, to generalised $f(R)$ theories of gravity. In view of the usefulness of this alternative framework to general relativity, its generalisation can be of potential importance for deriving analogous results to those obtained in general relativity. We generalise, as an example, the results of Maartens and Taylor to show that within the framework of general $f(R)$ theories, a perfect fluid spacetime with vanishing vorticity, shear and acceleration is Friedmann-Lemaitre-Robertson-Walker only if the fluid has in addition a barotropic equation of state. It then follows that the Ehlers-Geren-Sachs theorem and its "almost" extension also hold for $f(R)$ theories of gravity.
\end{abstract}

PACS number(s): 04.50.+h, 98.80.Hw, 98.80.Cq

\footnotetext{
*e-mail: S.F.Rippl@maths.qmw.ac.u

$\dagger$ e-mail: H.van.Elst@maths.qmw.ac.uk

‡e-mail: R.K.Tavakol@maths.qmw.ac.uk

$\S$ e-mail: TAYLOR@gauss.cam.wits.ac.za
} 


\section{Introduction}

Recently, Maartens and Taylor [13] examined the kinematical and dynamical properties of 4-D fluid spacetimes in quadratic gravity — with the gravitational Lagrangian density of the type

$$
\mathcal{L}_{G}=\frac{1}{2 \kappa_{0}} \sqrt{-g}\left[R+\alpha R^{2}+\beta R_{\mu \nu} R^{\mu \nu}\right],
$$

where $\alpha$ and $\beta$ are positive coupling constants, $R$ is the Ricci curvature scalar and $R_{\mu \nu}$ is the Ricci curvature tensor. They derived the generalisations of the equations governing relativistic fluid dynamics given by Ehlers [8] and Ellis [10] for general relativity (GR) to the quadratic case. The kinematic and dynamic formulation of fluid spacetimes has been extremely useful in studying a variety of problems within the context of GR and it is similarly expected to be of value in the case of higher-order theories of gravity, as it gives an alternative framework to study problems analogous to those considered in GR. An interesting example of this was given by Maartens and Taylor, when they showed that the usual kinematic characterisation of the Friedmann-Lemaitre-Robertson-Walker (FLRW) geometry no longer holds in the quadratic theory, but that it can be restored, if in addition the fluid is assumed to be barotropic. A consequence of this is that the Ehlers-Geren-Sachs theorem (EGS) [9] also holds in the setting of quadratic theories of gravity. The EGS theorem states that if a family of freely falling observers measure the self-gravitating background radiation to be everywhere exactly isotropic, then the Universe is exactly homogeneous. The importance of this theorem lies in the fact that the observable isotropy of the cosmic microwave background radiation (CMBR) may be employed" to deduce that the spacetime is also homogeneous and therefore of the FLRW type.

A shortcoming of the above work is that, as is customary, it confines itself to the quadratic theories, thereby implicitly assuming the quadratic theories to be the generic prototypes of the higher-order theories in general. Higher-order corrections to the action arise in attempts at generalising GR as for example in (i) the low energy limit of superstrings [6], (ii) in perturbation expansions of GR, where the existence

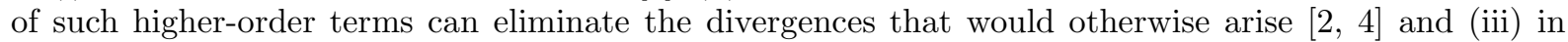
the renormalisation of higher-loop contributions which gives rise to terms in the action that are higher than quadratic. Such terms are thought to have been dominant at early epochs in the evolution of the Universe, when the curvature of spacetime was high.

This indicates that the genericity of the quadratic theories cannot be assumed a priori and higher than quadratic terms in the action are also likely to be involved in generalisations of GR. Further, there is no a priori reason why results obtained within the context of quadratic theories remain stable (in the sense of [7] ) with respect to cubic (and higher-order) perturbations to the action. In fact there are examples where the inclusion of cubic terms to the action can produce qualitatively important changes to the corresponding results obtained within the framework of the quadratic theories (see [11] for an example). To have a feel for this, recall that general higher-order theories with gravitational Lagrangian density of the form

$$
\mathcal{L}_{G}=\frac{1}{2 \kappa_{0}} \sqrt{-g} f(R),
$$

where $f(R)$ is a differentiable function of $R$ and $d f(R) / d R \neq 0$, can be expressed as GR plus a scalar field $\phi$, with a corresponding scalar potential $V(\phi)$ [3, 14. Now it turns out that the asymptotic behaviour of this potential depends crucially on the form of the action. For example, in 4-D spacetimes, the scalar potential $V(\phi)$ corresponding to quadratic theories tends asymptotically towards a finite constant limiting value, whereas for cubic and higher-order actions, it tends to zero [3]. Higher than quadratic terms in the action could therefore produce important consequences.

As a result, it is of importance to generalise the equations governing relativistic fluid dynamics given by Maartens and Taylor to the case of general higher-order gravity with gravitational Lagrangian density given by (2). This would be of value, as it allows an alternative framework within which general higher-order theories can be studied. Here as an example, we study the conditions for the kinematic characterisation of the FLRW models (and the EGS theorem), to see whether they are stable with respect to this action. We then use our results to test the stability of the almost-EGS theorem of [15, 17].

The plan of the paper is as follows. In section 2 we generalise the results of [13], by deriving the Ehlers-Ellis equations of relativistic fluid dynamics for a general action with a gravitational Lagrangian

\footnotetext{
${ }^{1}$ Assuming the isotropy to hold everywhere.
} 
density of the form (2) and a general energy-momentum tensor $T_{\mu \nu}$. In section 3 we consider the FLRW models, the EGS and almost-EGS theorems within the context of these theories, and finally section 4 contains the conclusion.

\section{Fluid Kinematics and Dynamics of $f(R)$ Theories}

In this section we generalise the equations of relativistic fluid dynamics given in [8, 10] to the case of generalised theories of gravity given by the action

$$
\mathcal{S}=\frac{1}{2 \kappa_{0}} \int_{\mathcal{M}} \sqrt{-g} f(R) d^{4} x+\int_{\mathcal{M}} \mathcal{L}_{M} d^{4} x
$$

where $f(R)$ is some arbitrary differentiable function of the Ricci curvature scalar, $\mathcal{L}_{M}$ is the Lagrangian density for the matter sources and we have chosen units such that $c=1$ and $\kappa_{0}=8 \pi G$. Throughout, Greek indices run from $\mu, \nu=0 \ldots 3$ and in a coordinate system $\left\{x^{0}, x^{a}\right\}(a=1,2,3)$, the spatial coordinates are denoted by Latin indices $a, b, \ldots$.

The variation of this action with respect to the metric tensor of spacetime, $g_{\mu \nu}$, results in the generalised field equations, given by

$$
\begin{aligned}
R_{\mu \nu}= & f^{\prime-1}\left[\frac{1}{2} f g_{\mu \nu}+f^{\prime \prime}\left(\nabla_{\mu} \nabla_{\nu} R-g_{\mu \nu} \square R\right)\right. \\
& \left.+f^{\prime \prime \prime}\left(\nabla_{\mu} R \nabla_{\nu} R-g_{\mu \nu} \nabla_{\rho} R \nabla^{\rho} R\right)+\kappa_{0} T_{\mu \nu}\right],
\end{aligned}
$$

where $f^{\prime}:=d f(R) / d R$, etc, $\square R:=g^{\mu \nu} \nabla_{\mu} \nabla_{\nu} R$, and the energy-momentum tensor of the matter sources is defined by the variational derivative $T^{\mu \nu}:=(2 / \sqrt{-g}) \delta \mathcal{L}_{M} / \delta g_{\mu \nu}$. (Note that $\nabla_{\mu} T^{\mu \nu}=0$ still holds [5].)

The contraction of this equation gives the Ricci curvature scalar in the form

$$
R=f^{\prime-1}\left[2 f-3 f^{\prime \prime} \square R-3 f^{\prime \prime \prime} \nabla_{\mu} R \nabla^{\mu} R+\kappa_{0} T\right],
$$

where $T$ denotes the trace of $T_{\mu \nu}$. Taking the covariant derivative of the Ricci scalar (5) one obtains

$$
\nabla_{\mu} R=\frac{3 f^{\prime \prime} \nabla_{\mu}(\square R)+6 f^{\prime \prime \prime}\left(\nabla_{\mu} \nabla_{\nu} R\right) \nabla^{\nu} R-\kappa_{0} \nabla_{\mu} T}{f^{\prime}-f^{\prime \prime} R-3 f^{\prime \prime \prime} \square R-3 f^{\prime \prime \prime \prime} \nabla_{\rho} R \nabla^{\rho} R} .
$$

Now following Ehlers [8] and Ellis [10], we define a projection tensor, which allows geometrical quantities to be projected into the instantaneous rest spaces of observers co-moving with the timelike matter flow with (normalised) 4 -velocity $u^{\mu}$, given by $h^{\mu}{ }_{\nu}:=\delta^{\mu}{ }_{\nu}+u^{\mu} u_{\nu}$. Projecting parallel and orthogonal to the matter flow lines one obtains a $1+3$ decomposition of the dynamic equations ("threading picture"). In particular this yields a decomposition of the covariant derivative of the matter 4 -velocity $u^{\mu}$, which is used to define kinematical quantities characteristic of the matter flow, in the form

$$
\nabla_{\nu} u_{\mu}:=-a_{\mu} u_{\nu}+\sigma_{(\mu \nu)}+\frac{1}{3} \Theta h_{\mu \nu}+\omega_{[\mu \nu]},
$$

where $a^{\mu}$ denotes the acceleration vector, $\sigma_{(\mu \nu)}$ the rate of shear tensor, $\Theta$ the rate of expansion scalar, and $\omega_{[\mu \nu]}$ the rate of vorticity tensor. Also in line with [8, 10], one can define the usual vorticity vector in the form $\left(\epsilon_{0123}=\sqrt{-g}\right) \omega^{\mu}:=\frac{1}{2} \epsilon^{\mu \nu \rho \sigma} \omega_{\nu \rho} u_{\sigma} \Rightarrow \omega_{\mu \nu}=\epsilon_{\mu \nu \rho \sigma} \omega^{\rho} u^{\sigma}$. by

The "electric" and "magnetic parts" of the trace-free Weyl conformal curvature tensor can be defined

$$
E_{(\mu \nu)}(u):=C_{\mu \rho \nu \sigma} u^{\rho} u^{\sigma}, \quad H_{(\mu \nu)}(u):=\frac{1}{2} \epsilon_{\mu \rho \alpha \beta} C_{\nu \sigma}^{\alpha \beta} u^{\rho} u^{\sigma} .
$$


Splitting the field equations as in the case of GR (and the quadratic case [13]), by contracting the Ricci tensor (四) with the 4 -velocity $u^{\mu}$ and the projection tensor $h^{\mu}{ }_{\nu}$, yields

$$
\begin{aligned}
R_{\mu \nu} u^{\mu} u^{\nu}= & f^{\prime-1}\left[-\frac{1}{2} f+f^{\prime \prime} h^{\mu \nu} \nabla_{\mu} \nabla_{\nu} R+f^{\prime \prime \prime} h^{\mu \nu} \nabla_{\mu} R \nabla_{\nu} R+\kappa_{0} T_{\mu \nu} u^{\mu} u^{\nu}\right] \\
R_{\nu \rho} h_{\mu}^{\nu} u^{\rho}= & f^{\prime-1}\left[f^{\prime \prime} h_{\mu}^{\nu}\left(\nabla_{\nu} \nabla_{\rho} R\right) u^{\rho}+f^{\prime \prime \prime} \dot{R} h^{\nu}{ }_{\mu} \nabla_{\nu} R+\kappa_{0} T_{\nu \rho} h_{\mu}^{\nu} u^{\rho}\right] \\
R_{\rho \sigma} h^{\rho}{ }_{\mu} h_{\nu}^{\sigma}= & f^{\prime-1}\left[\frac{1}{2} f h_{\mu \nu}+f^{\prime \prime} h^{\rho}{ }_{\mu} h_{\nu}^{\sigma}\left(\nabla_{\rho} \nabla_{\sigma} R-h_{\rho \sigma} \square R\right)\right. \\
& \left.+f^{\prime \prime \prime} h^{\rho}{ }_{\mu} h_{\nu}^{\sigma}\left(\nabla_{\rho} R \nabla_{\sigma} R-h_{\rho \sigma} \nabla_{\lambda} R \nabla^{\lambda} R\right)+\kappa_{0} T_{\rho \sigma} h_{\mu}^{\rho} h_{\nu}^{\sigma}\right] .
\end{aligned}
$$

As in the case of GR [8, 10], starting from the Ricci identities for the vector field $u^{\mu}$, multiplying by $u^{\nu}$ and projecting into the local 3-space, gives the propagation equation for $v_{\mu \nu}=h^{\alpha}{ }_{\mu} h^{\beta}{ }_{\nu} \nabla_{\alpha} u_{\beta}$, in the form

$$
h^{\alpha}{ }_{\mu} h^{\beta}{ }_{\nu} \dot{v}_{\alpha \beta}-a_{\mu} a_{\nu}-h^{\alpha}{ }_{\mu} h^{\beta}{ }_{\nu} \nabla_{\alpha} a_{\beta}+v^{\rho}{ }_{\mu} v_{\nu \rho}+R_{\alpha \gamma \beta \delta} h^{\alpha}{ }_{\mu} u^{\gamma} h^{\beta}{ }_{\nu} u^{\delta}=0 .
$$

The trace of this equation yields the generalised Raychaudhuri equation for the action (3)

$$
\begin{aligned}
0= & \dot{\Theta}+\frac{1}{3} \Theta^{2}-\nabla_{\mu} a^{\mu}+2\left(\sigma^{2}-\omega^{2}\right) \\
& +f^{\prime-1}\left[-\frac{1}{2} f+f^{\prime \prime} h^{\mu \nu} \nabla_{\mu} \nabla_{\nu} R+f^{\prime \prime \prime} h^{\mu \nu} \nabla_{\mu} R \nabla_{\nu} R+\kappa_{0} T_{\mu \nu} u^{\mu} u^{\nu}\right] .
\end{aligned}
$$

The skew part of (12) is the generalised vorticity propagation equation for the $f(R)$ theories,

$$
0=h_{[\mu}^{\alpha} h_{\nu]}^{\beta}\left(\dot{\omega}_{\alpha \beta}+\nabla_{\alpha} a_{\beta}\right)-2 \sigma_{[\mu}^{\rho} \omega_{\nu] \rho}+\frac{2}{3} \Theta \omega_{\mu \nu},
$$

which is identical to the Einstein case. On the other hand the symmetric, trace-free part of (12) is the shear propagation equation for the action (3) given by

$$
\begin{aligned}
0= & h_{(\mu}^{\alpha} h^{\beta}{ }_{\nu)}\left(\dot{\sigma}_{\alpha \beta}-\nabla_{\alpha} a_{\beta}\right)-a_{\mu} a_{\nu}+\omega_{\mu} \omega_{\nu}+\sigma_{\mu \rho} \sigma_{\nu}^{\rho}+\frac{2}{3} \Theta \sigma_{\mu \nu} \\
& +\frac{1}{3} h_{\mu \nu}\left(\nabla_{\rho} a^{\rho}-\omega^{2}-2 \sigma^{2}\right)+E_{\mu \nu} \\
& -\frac{1}{2} f^{\prime-1}\left(h^{\alpha}{ }_{\mu} h^{\beta}{ }_{\nu}-\frac{1}{3} h_{\mu \nu} h^{\alpha \beta}\right)\left[f^{\prime \prime} \nabla_{\alpha} \nabla_{\beta} R+f^{\prime \prime \prime} \nabla_{\alpha} R \nabla_{\beta} R+\kappa_{0} T_{\alpha \beta}\right] .
\end{aligned}
$$

One can also obtain the three constraint equations from the Ricci identities for $u^{\mu}$ in the form

$$
\begin{aligned}
0= & h^{\mu}{ }_{\nu}\left(\frac{2}{3} \nabla^{\nu} \Theta-h_{\sigma}^{\rho} \nabla_{\rho} \sigma^{\nu \sigma}\right)+\epsilon^{\mu \nu \rho \sigma} u_{\sigma}\left(\nabla_{\nu} \omega_{\rho}-2 \omega_{\nu} a_{\rho}\right) \\
& +f^{\prime-1}\left[f^{\prime \prime} h^{\mu}{ }_{\nu}\left(\nabla^{\nu} \nabla_{\rho} R\right) u^{\rho}+f^{\prime \prime \prime} \dot{R} h^{\mu}{ }_{\nu} \nabla^{\nu} R+\kappa_{0} T^{\nu}{ }_{\rho} h^{\mu}{ }_{\nu} u^{\rho}\right],
\end{aligned}
$$

which are the field equations (10), together with

$$
\begin{gathered}
h^{\mu}{ }_{\nu} \nabla_{\mu} \omega^{\nu}=a_{\mu} \omega^{\mu} \\
H_{\mu \nu}=2 a_{(\mu} \omega_{\nu)}+h_{(\mu}^{\alpha} h^{\beta}{ }_{\nu)}\left(\nabla^{\gamma} \omega_{\alpha}{ }^{\delta}+\nabla^{\gamma} \sigma_{\alpha}{ }^{\delta}\right) \epsilon_{\beta \gamma \delta \epsilon} u^{\epsilon} .
\end{gathered}
$$

Using the decomposition of the Riemann curvature tensor into the trace-free Weyl conformal curvature tensor, the Ricci curvature tensor and the Ricci curvature scalar,

$$
R_{\mu \nu \rho \sigma}:=C_{\mu \nu \rho \sigma}+g_{\mu[\rho} R_{\sigma] \nu}+g_{\nu[\sigma} R_{\rho] \mu}-\frac{1}{3} R g_{\mu[\rho} g_{\sigma] \nu},
$$

the Bianchi identities

$$
\nabla_{[\mu} R_{\nu \rho] \sigma \tau}=0
$$

can be cast into the form 12

$$
\nabla_{\sigma} C^{\mu \nu \rho \sigma}=-\nabla^{[\mu} R^{\nu] \rho}-\frac{1}{6} g^{\rho[\mu} \nabla^{\nu]} R
$$


Now using equations (6)-(11), the constraint and evolution equations for $E_{\mu \nu}$ and $H_{\mu \nu}$ can be derived for the case of $f(R)$ theories, in the form

$$
\begin{aligned}
& h^{\mu}{ }_{\alpha} h_{\beta}^{\gamma} \nabla_{\gamma} E^{\alpha \beta}=\epsilon^{\mu \alpha \beta \gamma} \sigma_{\alpha \delta} H^{\delta}{ }_{\beta} u_{\gamma}-3 H^{\mu}{ }_{\alpha} \omega^{\alpha} \\
& +\kappa_{0} f^{\prime-1} h_{\alpha}^{\mu}\left[h_{\gamma}^{\beta} \nabla^{[\alpha} T_{\beta}^{\gamma]}-\frac{1}{3} \nabla^{\alpha} T\right] \\
& +\frac{1}{2} f^{\prime-1} f^{\prime \prime} h^{\mu}{ }_{\alpha}\left[\dot{R} R^{\alpha}{ }_{\beta} u^{\beta}-\left(R_{\beta \gamma} u^{\beta} u^{\gamma}+\frac{1}{3} R\right) \nabla^{\alpha} R\right. \\
& \left.-\nabla^{\alpha}(\square R)-\left(\nabla^{\alpha} \nabla_{\beta} R\right)^{\cdot} u^{\beta}+h_{\gamma}^{\beta} \nabla^{\alpha}\left(\nabla_{\beta} \nabla^{\gamma} R\right)\right] \text {, } \\
& h^{\mu}{ }_{\alpha} h^{\nu} \dot{E}^{\alpha \beta}=3 E^{(\mu}{ }_{\alpha} \sigma^{\nu) \alpha}+E^{(\mu}{ }_{\alpha} \omega^{\nu) \alpha}-\Theta E^{\mu \nu}-h^{\mu \nu} E_{\alpha \beta} \sigma^{\alpha \beta} \\
& -2 H_{\alpha}^{(\mu} \epsilon^{\nu) \alpha \beta \gamma} a_{\beta} u_{\gamma}+h^{(\mu}{ }_{\alpha} \epsilon^{\nu) \beta \gamma \delta} \nabla_{\beta} H_{\gamma}^{\alpha} u_{\delta} \\
& +\frac{\kappa_{0}}{2} f^{\prime-1}\left[h_{\alpha}^{(\mu} h_{\beta}^{\nu)} \nabla^{\alpha} T_{\gamma}^{\beta} u^{\gamma}-h_{\alpha}^{(\mu} h_{\beta}^{\nu)_{\beta}}\left(T^{\alpha \beta}\right)^{\cdot}+\frac{1}{3} \dot{T} h^{\mu \nu}\right] \\
& +\frac{1}{2} f^{\prime-1} f^{\prime \prime} h_{\alpha}^{(\mu} h_{\beta}^{\nu)_{\beta}}\left[\dot{R}\left(R^{\alpha \beta}-\frac{1}{3} R g^{\alpha \beta}\right)-\nabla^{\alpha} R R_{\gamma}^{\beta} u^{\gamma}\right. \\
& \left.+\nabla^{\alpha}\left(\nabla_{\gamma} \nabla^{\beta} R\right) u^{\gamma}-\left(\nabla^{\alpha} \nabla^{\beta} R\right)^{\cdot}\right] \text {, } \\
& h^{\mu}{ }_{\alpha} h_{\beta}^{\gamma} \nabla_{\gamma} H^{\alpha \beta}=-\epsilon^{\mu \alpha \beta \gamma} \sigma_{\alpha \delta} E^{\delta}{ }_{\beta} u_{\gamma}+3 E^{\mu}{ }_{\alpha} \omega^{\alpha} \\
& +\frac{\kappa_{0}}{2} f^{\prime-1} \epsilon^{\mu \alpha \beta \gamma} \nabla_{\alpha} T_{\beta}^{\delta} u_{\gamma} u_{\delta} \\
& +\frac{1}{2} f^{\prime-1} f^{\prime \prime} \epsilon^{\mu \alpha \beta \gamma}\left[\nabla_{\alpha} \nabla_{\beta} \nabla^{\delta} R-\nabla_{\alpha} R R_{\beta}^{\delta}\right] u_{\gamma} u_{\delta}, \\
& h^{\mu}{ }_{\alpha} h_{\beta}^{\nu} \dot{H}^{\alpha \beta}=3 H^{(\mu}{ }_{\alpha} \sigma^{\nu) \alpha}+H^{(\mu}{ }_{\alpha} \omega^{\nu) \alpha}-\Theta H^{\mu \nu}-h^{\mu \nu} H_{\alpha \beta} \sigma^{\alpha \beta} \\
& +2 E^{(\mu}{ }_{\alpha} \epsilon^{\nu) \alpha \beta \gamma} a_{\beta} u_{\gamma}-h_{\alpha}^{(\mu} \epsilon^{\nu) \beta \gamma \delta} \nabla_{\beta} E_{\gamma}^{\alpha} u_{\delta} \\
& +\frac{\kappa_{0}}{2} f^{\prime-1} h_{\alpha}^{(\mu} \epsilon^{\nu) \beta \gamma \delta} \nabla_{\beta} T_{\gamma}^{\alpha} u_{\delta} \\
& +\frac{1}{2} f^{\prime-1} f^{\prime \prime} h_{\alpha}^{(\mu} \epsilon^{\nu) \beta \gamma \delta}\left[\nabla_{\beta} R R_{\gamma}^{\alpha}-\nabla_{\beta} \nabla_{\gamma} \nabla^{\alpha} R\right] u_{\delta} .
\end{aligned}
$$

These equations generalise the analogous equations given for the quadratic Lagrangian theories of gravity [13] to the $f(R)$ case with a general energy-momentum tensor $T_{\mu \nu}$.

And finally we derive, by use of the Gauß equation, which holds for spacetime configurations with a hypersurface-orthogonal matter fluid flow $\left(\omega_{\mu \nu}=0\right)$, the 3-Ricci curvature tensor of the spacelike 3 -surfaces orthogonal to $u^{\mu}$. The 3 -Riemann curvature tensor ${ }^{3} R_{\mu \nu \rho \sigma}$ is given by

$$
{ }^{3} R_{\mu \nu \rho \sigma}={ }^{3} R_{\mu \rho} h_{\nu \sigma}-{ }^{3} R_{\mu \sigma} h_{\nu \rho}+{ }^{3} R_{\nu \sigma} h_{\mu \rho}-{ }^{3} R_{\nu \rho} h_{\mu \sigma}-\frac{1}{2}{ }^{3} R\left(h_{\mu \rho} h_{\nu \sigma}-h_{\mu \sigma} h_{\nu \rho}\right) .
$$

Using the field equations (11), we can split the 3-Ricci tensor ${ }^{3} R_{\mu \nu}$ into its trace

$$
\begin{aligned}
{ }^{3} R= & 2 \sigma^{2}-\frac{2}{3} \Theta^{2} \\
& +f^{\prime-1}\left[f+f^{\prime \prime}\left(2 h^{\mu \nu} \nabla_{\mu} \nabla_{\nu} R-3 \square R\right)+f^{\prime \prime \prime}\left(2 \dot{R}^{2}-\nabla_{\mu} \nabla^{\mu} R\right)+\kappa_{0}\left(2 T_{\mu \nu} u^{\mu} u^{\nu}+T\right)\right],
\end{aligned}
$$

and its trace-free part

$$
\begin{aligned}
{ }^{3} R_{\mu \nu}-\frac{1}{3}{ }^{3} R h_{\mu \nu}= & h_{(\mu}^{\alpha} h^{\beta}{ }_{\nu)}\left(\nabla_{\alpha} a_{\beta}-\dot{\sigma}_{\alpha \beta}\right)-\Theta \sigma_{\mu \nu}+a_{\mu} a_{\nu}-\frac{1}{3} h_{\mu \nu} \nabla_{\rho} a^{\rho} \\
& +f^{\prime-1}\left(h^{\alpha}{ }_{\mu} h^{\beta}{ }_{\nu}-\frac{1}{3} h_{\mu \nu} h^{\alpha \beta}\right)\left[f^{\prime \prime} \nabla_{\alpha} \nabla_{\beta} R+f^{\prime \prime \prime} \nabla_{\alpha} R \nabla_{\beta} R+\kappa_{0} T_{\alpha \beta}\right] .
\end{aligned}
$$

These relations then complete the generalisations of the kinematical and the dynamical equations given by Ehlers and Ellis [8, 10] for GR and those given by [13] for the quadratic gravity to the case of $f(R)$ theories with a general energy-momentum tensor $T_{\mu \nu}$.

\section{FLRW Cosmological Models in $f(R)$ Theories}

In this section we look for conditions for the kinematic characterisation of FLRW geometry in the context of $f(R)$ theories. To begin with let us recall that the kinematic conditions, in the context of GR and for perfect fluid spacetimes, are given by

$$
a_{\mu}=\omega_{\mu \nu}=\sigma_{\mu \nu}=0
$$


which with coordinates $x^{\alpha}=\left(t, x^{a}\right)$ allow the metric to be expressible in the following form

$$
d s^{2}=-d t^{2}+A(t)^{2} \Lambda_{a b}\left(x^{c}\right) d x^{a} d x^{b},
$$

such that the spacelike 3 -surfaces defined by the metric $\Lambda_{a b}\left(x^{c}\right)$ have constant curvature. In usual Einstein's general relativity, the conditions (29) along with the constraint equations (16) - (18) imply the vanishing of the Weyl tensor. However, for the $f(R)$ generalised equations with perfect fluid matter source, which we shall assume here, only $H_{\mu \nu}$ is immediately zero, and therefore to show that $E_{\mu \nu}=0$ we need to show that the Ricci scalar is spatially homogeneous. To do this, we recall [1] that the kinematic conditions (29) imply the existence of the metric

$$
d s^{2}=-d t^{2}+S\left(t, x^{c}\right)^{2} \lambda_{a b}\left(x^{c}\right) d x^{a} d x^{b} \quad u^{\mu}=\delta_{0}^{\mu},
$$

where $\frac{1}{3} \Theta=\nabla_{0} S / S$. The (0a) field equations (10) together with the constraint equations (16) imply that

$$
\partial_{a} \partial_{0}(\ln S)=\frac{1}{3} \nabla_{a} \Theta=-\frac{1}{2 f^{\prime}} \nabla_{a} \nabla_{0}\left(f^{\prime}\right) .
$$

Now, if we assume that our matter fluid source obeys a barotropic equation of state of the form $p=$ $p(\rho), \quad d p(\rho) / d \rho \neq 0$, where $p$ and $\rho$ denote the isotropic pressure and the total energy density of the matter fluid respectively, then the conservation equations $\nabla_{\nu} T^{\mu \nu}=0$ with the kinematic conditions (29) imply that

$$
h^{\nu}{ }_{\mu} \nabla_{\nu} p=h^{\nu}{ }_{\mu} \nabla_{\nu} \rho=h^{\nu}{ }_{\mu} \nabla_{\nu} \Theta=0 .
$$

In the coordinates of (31), (33) gives $\Theta=\Theta(t)$, therefore, (32) implies $S=A(t) B\left(x^{a}\right)$, which reduces the metric (31) to the form (30).

The next step is to show that $\Lambda_{a b}\left(x^{c}\right)$ has constant curvature. Again, from (32) and (33) we have $\nabla_{a} \nabla_{0}\left(f^{\prime}\right)=0$ which, using the connection derived from (30), integrates to

$$
f^{\prime}=A(t) g\left(x^{a}\right)+B(t),
$$

where $g$ and $B$ are arbitrary functions of $x^{a}$ and $t$ respectively. To proceed from here is not as straightforward as the proof in [13. We use equations (5) and (9) to eliminate the term involving $f$. The resulting equation, using (30), (31) and the perfect fluid form of the energy-momentum tensor is

$$
\frac{6 \dot{A}^{2}}{A^{2}}-\frac{6 \ddot{A}}{A}+\frac{3}{A^{2}}=\frac{1}{f^{\prime}}\left[\frac{1}{A^{2}} \Lambda^{a b} \nabla_{a} \nabla_{b}\left(f^{\prime}\right)+3 \nabla_{0} \nabla_{0}\left(f^{\prime}\right)+3 \kappa_{0}(\rho+p)\right],
$$

where ${ }^{3} R^{*}\left(x^{c}\right)$ is the 3 -Ricci scalar of the spacelike 3 -surfaces defined by the metric $\Lambda_{a b}\left(x^{c}\right)$. Using equation (34) and the form of the metric (30), equation (35) can be reduced to (provided $A \neq 0$ )

$$
h\left(x^{a}\right)+B(t)^{3} R^{*}\left(x^{a}\right)+9 \dot{A}(t)^{2} g\left(x^{a}\right)+C(t)=0
$$

where $h\left(x^{a}\right)$ and $C(t)$ are given functions and $A, B$ and $g$ are defined by (30) and (34). It is clear from equation (36) that $\nabla_{a} g=0$ and consequently that $\nabla_{a}\left(f^{\prime}\right)=0$, which in turn implies

$$
h^{\nu}{ }_{\mu} \nabla_{\nu} R=0 \text {. }
$$

Now to show that $\Lambda_{a b}\left(x^{c}\right)$ has constant curvature we proceed as follows. Equation (37) and the (GR) FLRW kinematic conditions (29) imply [1]

$$
\nabla_{\mu} \nabla_{\nu} R=\ddot{R} u_{\mu} u_{\nu}-(\dot{R} \dot{A} / A) h_{\mu \nu}
$$

which by (15) and (28) implies

$$
E_{\mu \nu}=0={ }^{3} R_{\mu \nu}-\frac{1}{3}{ }^{3} R h_{\mu \nu},
$$

where ${ }^{3} R$ is the 3 -Ricci tensor formed from $h_{a b}\left(t, x^{c}\right)$, proving that the spacelike 3 -surfaces $t=$ constant are intrinsically isotropic. And finally we need to show that such 3 -surfaces are isotropically embedded. The reasoning for this remains unchanged to the one given in [13] for the quadratic theories. First of all recall that 16

$$
R_{a b}={ }^{3} R_{a b}^{*}\left(x^{c}\right)+\left(A \ddot{A}+2 \dot{A}^{2}\right) \Lambda_{a b},
$$


where ${ }^{3} R^{*}{ }_{a b}\left(x^{c}\right)$ is the 3 -Ricci tensor formed from $\Lambda_{a b}\left(x^{c}\right)$. It can also be shown, using the field equation (11) together with (9) and (5), that

$$
R_{a b}=\left(\frac{1}{3} A^{2} R-A \ddot{A}\right) \Lambda_{a b} .
$$

Now since $A$ and $R$ are functions of $t$ only, equations (40) and (41) imply that ${ }^{3} R^{*}{ }_{a b}\left(x^{c}\right)=2 K \Lambda_{a b}\left(x^{c}\right)$, where

$$
2 K=\frac{1}{3} A^{2} R-2 A \ddot{A}-2 \dot{A}^{2}=\text { constant, }
$$

and $K$ is the constant curvature of $\Lambda_{a b}\left(x^{c}\right)$. This then shows that the spacelike 3 -surfaces $t=$ constant are isotropic and isotropically embedded and therefore the metric is FLRW. The corresponding Bianchi identities (22) - (25) are all identically satisfied, except for the $E_{\mu \nu}$ propagation equation (23), which reduces to the field equation (11).

In this way we have a generalisation of the result given in [13], thus:

In the general $f(R)$ theories of gravity, a perfect fluid spacetime with vanishing vorticity, shear and acceleration is FLRW only if the fluid has in addition a barotropic equation of state.

An important consequence of this result is that the Ehlers-Geren-Sachs theorem also holds for the $f(R)$ theories of gravity.

In this sense then, the main conclusions of Maartens and Taylor remain stable to any perturbations of the action. This represents a significant factor in favour of the higher-order theories given that the EGS theorem is one of the key results motivating the standard big-bang model of the Universe.

Finally, it has recently been shown [15] that the observed almost-isotropy of the cosmic microwave background radiation (CMBR) implies that the Universe has been almost spatially homogeneous and isotropic since the photon decoupling 2. In terms of covariant perturbation theory [15], this means that deviations of the spacetime kinematics and dynamics from FLRW are at most of order one with respect to some smallness parameter $\epsilon$. In other words, if a quantity disappears in a FLRW spacetime, it is of order one (or higher) in an almost-FLRW spacetime, which allows the metric to be written in a perturbed FLRW form [15]. Subsequently, Taylor and Maartens [17] have shown that this theorem also holds in quadratic theories of gravity and may have applications to the very early Universe.

Now the results of the section 2, together with the above result that EGS theorem also holds for the general $f(R)$ theories of gravity, imply that the almost-EGS theorem shown to hold for quadratic gravity of [17] is further generalisable to the case of $f(R)$ theories. We see this as follows.

We use the formalism of [15, 17] and elaborate only on the deviations from the proof of [17] where necessary. We consider an acceleration-free $\left(a^{\mu}=0\right)$ expanding congruence $(\Theta>0)$, where the kinematics is determined by the shear, vorticity and expansion. It can be shown in a manner analogous to that in [15], that the components of the energy-momentum tensor, which deviate from perfect fluid form, are not dissipative quantities. Then the energy flux and the anisotropic stress, $q_{\mu}$ and $\pi_{\mu \nu}$, measure the deviation of the distribution function from isotropy and, since they vanish in FLRW spacetimes, are first order quantities, i.e.

$$
q_{\mu}, \pi_{\mu \nu}=O[1] .
$$

From the conservation equations it is possible to derive the kinematic conditions (29) to first order, giving

$$
\omega_{\mu \nu}, \sigma_{\mu \nu}=O[1] \text {. }
$$

Furthermore, it is also possible to establish the conditions (33) to first order:

$$
h^{\nu}{ }_{\mu} \nabla_{\nu} p, h^{\nu}{ }_{\mu} \nabla_{\nu} \rho, h^{\nu}{ }_{\mu} \nabla_{\nu} \Theta=O[1] .
$$

Equations (43), (44) and (45) are crucial to proving almost-homogeneity of the scalar curvature. From the constraint equations $(16)$ we have that

$$
h^{\mu}{ }_{\nu}\left(\nabla^{\nu} \nabla_{\rho} f^{\prime}\right) u^{\rho}=O[1] .
$$

${ }^{2}$ This amounts to an almost EGS theorem. 
By taking the spatial derivative of the generalised Raychaudhuri equation (13) and using (46) we obtain

$$
\left[f^{\prime \prime}\left(8 \dot{\Theta}+2 \Theta^{2}-2 R\right)-f^{\prime}\right] h^{\nu}{ }_{\mu} \nabla_{\nu} R=O[1]
$$

which in view of the fact that $R, \Theta$ and $\dot{\Theta}$ are non-zero in the exactly isotropic (FLRW) case, they would be $O[0]$ and we can deduce

$$
h^{\nu}{ }_{\mu} \nabla_{\nu} R=O[1]
$$

Then from (15) and (18) we obtain

$$
E_{\mu \nu}, H_{\mu \nu}=O[1]
$$

which establishes the almost-FLRW kinematics to $O[1]$. Equation (48) implies also that the 3-surfaces of constant energy density are almost-isotropic and almost-isotropically embedded. We see this from equations (28) and (42), where it follows from (48) that

$$
{ }^{3} R_{\mu \nu}-\frac{1}{3}{ }^{3} R h_{\mu \nu}=O[1]
$$

and

$$
2 K=\frac{1}{3} A^{2} R-2 A \ddot{A}-2 \dot{A}^{2} \quad, \quad \dot{K}=O[1] .
$$

Thus, it is possible to recover all the standard relations governing an FLRW model of the Universe to $O[0]$, and in particular, to show that there exists an almost-FLRW metric in general $f(R)$ gravity. This then shows that the "almost" EGS generalises to the case of $f(R)$ theories.

\section{Conclusion}

We have derived the generalised kinematic and dynamic equations governing relativistic fluid dynamics (given by Ehlers and Ellis for GR and for the quadratic theories by Maartens and Taylor) in the case of generalised $f(R)$ theories of gravity and with a general $T_{\mu \nu}$. Such generalisation is of potential importance as it gives an alternative framework in order to derive results analogous to those obtained in GR for the generalised theories of gravity.

As an example of application of these results, we have generalised the results of Maartens and Taylor to show that within the framework of $f(R)$ theories a perfect fluid spacetime with vanishing vorticity, shear and acceleration is FLRW only if the fluid has in addition a barotropic equation of state. It then follows that the Ehlers-Geren-Sachs theorem holds for general $f(R)$ theories as well. In this sense, then, the results of Maartens and Taylor are stable to perturbations of any order in the action. We also show that the "almost" EGS theorem of Stoeger, Maartens and Ellis and its generalisation derived by Taylor and Maartens in the case of quadratic gravity also remains stable to perturbations to the Lagrangian of the form given in equation (3).

\section{Acknowledgements}

We would like to thank Roy Maartens for many helpful discussions. SR is supported by a PPARC studentship, HvE is supported by a grant from the Drapers' Society at QMW and RT is supported by the PPARC UK, under grant No. H09454. DT thanks David Matravers and Portsmouth University for hospitality and a research grant and Astronomy Unit, QMW for visiting support. 


\section{References}

[1] Anderson, J M 1967, Principles of Relativity Physics, Academic Press, New York

[2] Antoniadis I and Tomboulis E T 1986, 'Gauge Invariance and Unitarity in Higher-Derivative Quantum Gravity', Phys. Rev. D 33, 2756

[3] Barrow J D and Cotsakis S 1988, 'Inflation and the Conformal Structure of Higher-Order Gravity Theories', Phys. Letts. 214B, 515

[4] Barth N H and Christensen S M 1983, 'Quantizing Fourth-Order Gravity Theories: The Functional Integral', Phys. Rev. D 28, 1876

[5] Buchdahl H A 1970, 'Non-Linear Lagrangians and Cosmological Theory', Mon. Not. R. Astron. Soc. 150,1

[6] Candelas P, Horowitz G T, Strominger A and Witten E 1985, 'Vacuum Configurations for Superstrings', Nucl. Phys. B258, 46

[7] Coley A A, Tavakol R K 1992, 'Fragility in Cosmology', Gen. Rel. Grav. 24, 835

[8] Ehlers J 1961, 'Beiträge zur relativistischen Mechanik kontinuierlicher Medien', Akad. Wiss. Lit. Mainz, Abhandl. Math.-Nat. Kl. 11

[9] Ehlers J, Geren P and Sachs R K 1968, 'Isotropic Solutions of the Einstein-Liouville Equations', J. Math. Phys. 9, 1344

[10] Ellis G F R 1971, 'Relativistic Cosmology', in General Relativity and Cosmology, Proceedings of the XLVII Enrico Fermi Summer School, Ed. R K Sachs, Academic Press, New York

[11] van Elst H, Lidsey J E and Tavakol R 1994, 'Quantum Cosmology and Higher-Order Lagrangian Theories', Class. Quantum Grav. 11, 2483

[12] Kundt W, Trümper M 1962, 'Beiträge zur Theorie der Gravitations-Strahlungsfelder', Akad. Wiss. Lit. Mainz, Abhandl. Math.-Nat. Kl. 12

[13] Maartens R, Taylor D R 1994, 'Fluid Dynamics in Higher Order Gravity', Gen. Rel. Grav. 26, 599

[14] Maeda K 1989, 'Towards the Einstein-Hilbert Action via Conformal Transformation', Phys. Rev. D 39, 3159

[15] Stoeger W R, Maartens R, Ellis G F R 1995, 'Proving Almost-Homogeneity of the Universe: An Almost Ehlers-Geren-Sachs Theorem', Astrophys. J. 443, 1

[16] Stephani H 1990, General Relativity, Cambridge University Press, Cambridge.

[17] Taylor D R, Maartens R 1995, 'Almost-Homogeneity of the Universe in Higher-Order Gravity', Portsmouth University Preprint, RCG 95/6 